\title{
Mesenchymal stromal cells shape the MDS microenvironment by inducing suppressive monocytes that dampen NK cell function
}

\author{
Dhifaf Sarhan, ${ }^{1,2}$ Jinhua Wang, ${ }^{3,4}$ Upasana Sunil Arvindam, ${ }^{3}$ Caroline Hallstrom, ${ }^{3}$ \\ Michael R. Verneris, ${ }^{5}$ Bartosz Grzywacz, ${ }^{6}$ Erica Warlick,, ${ }^{1}$ Bruce R. Blazar, ${ }^{7}$ and Jeffrey S. Miller ${ }^{1,3}$ \\ 'Department of Medicine, Division of Hematology, Oncology, and Transplantation, Masonic Cancer Center, University of \\ Minnesota, Minneapolis, Minnesota, USA. ${ }^{2}$ Department of Microbiology, Tumor and Cell Biology, Karolinska Institutet, \\ Stockholm, Sweden. ${ }^{3}$ Masonic Cancer Center and ${ }^{4}$ Institute of Health Informatics, University of Minnesota, Minneapolis, \\ Minnesota, USA. ${ }^{5}$ Pediatric Bone Marrow Transplantation, University of Colorado, Aurora, Colorado, USA. ${ }^{6}$ Department \\ of Laboratory Medicine and Pathology and 'Department of Pediatrics, Division of Blood and Marrow Transplantation and \\ Masonic Cancer Center, University of Minnesota, Minneapolis, Minnesota, USA.
}

\begin{abstract}
Altered BM hematopoiesis and immune suppression are hallmarks of myelodysplastic syndrome (MDS). While the BM microenvironment influences malignant hematopoiesis, the mechanism leading to MDS-associated immune suppression is unknown. We tested whether mesenchymal stromal cells (MSCs) contribute to this process. Here, we developed a model to study cultured MSCs from patients with MDS (MDS-MSCs) compared with those from aged-matched normal controls for regulation of immune function. MDS-MSCs and healthy donor MSCs (HD-MSCs) exhibited a similar in vitro phenotype, and neither had a direct effect on NK cell function. However, when MDS- and HD-MSCs were cultured with monocytes, only the MDS-MSCs acquired phenotypic and metabolic properties of myeloid-derived suppressor cells (MDSCs), with resulting suppression of NK cell function, along with T cell proliferation. A MSC transcriptome was observed in MDS-MSCs compared with HD-MSCs, including increased expression of the ROS regulator, ENC1. High ENC1 expression in MDS-MSCs induced suppressive monocytes with increased INHBA, a gene that encodes for a member of the TCF- $\beta$ superfamily of proteins. These monocytes also had reduced expression of the TCF- $\beta$ transcriptional repressor MAB21L2, further adding to their immunesuppressive function. Silencing ENC1 or inhibiting ROS production in MDS-MSCs abrogated the suppressive function of MDS-MSC-conditioned monocytes. In addition, silencing MAB21L2 in healthy MSC-conditioned monocytes mimicked the MDS-MSC-suppressive transformation of monocytes. Our data demonstrate that MDS-MSCs are responsible for inducing an immunesuppressive microenvironment in MDS through an indirect mechanism involving monocytes.
\end{abstract}

Conflict of interest: The authors have declared that no conflict of interest exists.

Copyright: ( 2020 , American Society for Clinical Investigation.

Submitted: May 9, 2019

Accepted: February 6, 2020

Published: March 12, 2020.

Reference information: /CI Insight. 2020;5(5):e130155.

https://doi.org/10.1172/jici.

insight.130155.

\section{Introduction}

Myelodysplastic syndrome (MDS) is a clonal malignant disease of the hematopoietic stem cell. The condition occurs most frequently in elderly individuals ( $85 \% \geq 60$ years). MDS is characterized by multilineage cytopenia and ineffective hematopoiesis, often with increased blast production, resulting in BM failure and a risk of transformation to acute myelogenous leukemia (AML) $(1,2)$. In the MDS microenvironment, the stromal cell compartment, including mesenchymal stromal cells (MSCs), contributes to an altered BM microenvironment, with dysregulation in proinflammatory cytokines and induction of hypoxia, leading to abnormalities in supportive hematopoietic niches (3). However, the role of MSCs in BM remains bidirectional, as they may directly alter the proliferation of CD34 hematopoietic stem cells and influence the BM immune compartments. Conversely, they may activate the innate and adaptive immune system $(4,5)$. Studies have shown that MSCs undergo molecular and genetic changes contributing to disease progression (6). Other studies failed to show functional differences between MSCs from patients with MDS (MDS-MSCs) and healthy individuals $(7,8)$. 
MSCs are multipotent cells that are capable of self-renewal and give rise to cell types, including osteoblasts, adipocytes, chondrocytes (9), endothelial cells (10), cardiomyocytes (11), and hepatocytes (12), allowing them to migrate and reside in various organs. MSCs participate in immunomodulation and immune dysregulation in the tumor microenvironment (13), interacting with various immune cell types, including T cells, NK cells, and myeloid cells. This interaction promotes direct cell-to-cell contact regulation as well as the production of soluble mediators. MSCs are able to impair DC antigen presentation by inhibiting the expression of MHC I and II molecules and costimulatory proteins, such as CD83, and IL-12 production $(14,15)$. MSCs induce anergy of activated T cells as a result of IL-10 production that enhances the development of regulatory $\mathrm{T}$ cells, thus inhibiting conventional $\mathrm{T}$ cell proliferation and other effector cell activities. In addition, MSCs derived from BM of healthy donors (HDs) impair the cytolytic activity of resting NK cells through production of indoleamine 2,3-dioxygenase (IDO) and prostaglandin E2 (PGE2) (16). MSC-mediated immunosuppression may be partially explained by soluble factors secreted by MSCs, including known immunosuppressants, such as IL-10, PGE2, NO, IDO, and TGF- $\beta$ (16-20).

In this study, we investigated mechanisms initiating MDS-associated immune suppression by studying MSC interactions with NK cells, T cells, and monocytes, using MDS-MSCs as compared with those from patients with healthy BM undergoing hip replacement surgery. We found that MDS-MSCs exhibited altered ENC1 expression, resulting in a conversion of monocytes to myeloid suppressor cells, to suppress $\mathrm{NK}$ and $\mathrm{T}$ cell function.

\section{Results}

MSC phenotype and function. Previous studies have defined an array of markers to characterize MSCs (21). Here, we used BM-derived MDS-MSCs compared with those derived from patients with otherwise healthy BM undergoing hip replacement as a suitable age-matched control group (median age, $66 \pm 18$ vs. $60 \pm$ 12 years). Cells were assessed for phenotype and potential to differentiate toward osteoblasts and adipocytes to confirm their MSC characteristics. We found that both HD-MSCs and MDS-MSCs were adherent to plastic and expressed the MSC markers CD73, CD90, CD105, and CD44 and lacked expression of the hematopoietic cell markers CD34 and MHCII (HLA-DR) (Figure 1A). MSCs derived from HDs and patients with MDS exhibited differentiation into osteoblasts and adipocytes (Supplemental Figure 1A; supplemental material available online with this article; https://doi.org/10.1172/jci.insight.130155DS1) and proliferated to a similar degree (Supplemental Figure 1B).

To determine whether MDS-MSCs have immunoregulatory effects on NK cells, HD-MSCs and MDSMSCs were cultured in a direct cell-to-cell culture with allogeneic NK cells from HDs. After 5 days of culture with IL-15, NK cell function was evaluated following a 6-hour stimulation with IL-12 and IL-18 to induce IFN- $\gamma$ production and an agonistic anti-CD16 antibody for degranulation. NK cell degranulation, IFN- $\gamma$ production, and proliferation were not different in cultures with MDS-MSCs compared with NK cells cultured alone or with HD-MSCs (Figure 1B).

MDS-MSCs induce immune-suppressive monocytes. Given evidence that MSCs modulate the BM environment in health and disease $(22,23)$, we investigated whether MDS-MSCs might regulate monocytes to indirectly modulate immune function. Purified blood monocytes were supplemented with low-dose GM-CSF and cultured alone for 7 days (7-day control monocytes) or with the addition of HD-MSCs or MDS-MSCs (7-day MSC-conditioned monocytes). Seven-day MDS-MSC but not 7-day control or 7-day HD-MSC-conditioned monocytes exhibited an immune-suppressive phenotype resembling monocytic myeloid-derived suppressor cells (MDSCs) positive for CD33 and CD14, with downregulation of HLA-DR and elevated expression of PD-L1 (Figure 2A). There was a modest elevation in CXCR5 and PVR (CD155) that did not reach statistical significance, no change in viability (Supplemental Figure 1C), and no change in CD11b, CXCR1, CXCR2, CXCR3, CXCR4, and nectin2 (data not shown).

An increased number of MDSCs has been observed in the tumor microenvironment of patients with MDS (24). These MDSCs have higher glycolytic capacity and increased mitochondrial activity (25). Thus, we examined the metabolic changes of monocytes following conditioning with MSCs. Seven-day MDSMSC-conditioned monocytes had increased metabolic activity shown by significant increases in oxygen consumption rate (OCR), accompanied by higher extracellular acidification rates (ECARs), reflecting an acceleration in mitochondrial respiration and glycolysis similar to in vitro-generated MDSCs (Figure 2B). Seven-day HD-MSC-conditioned monocytes had comparable levels of OCR and ECAR to 7-day control monocytes. OCR and ECAR levels were markedly lower compared with in vitro-generated MDSCs 

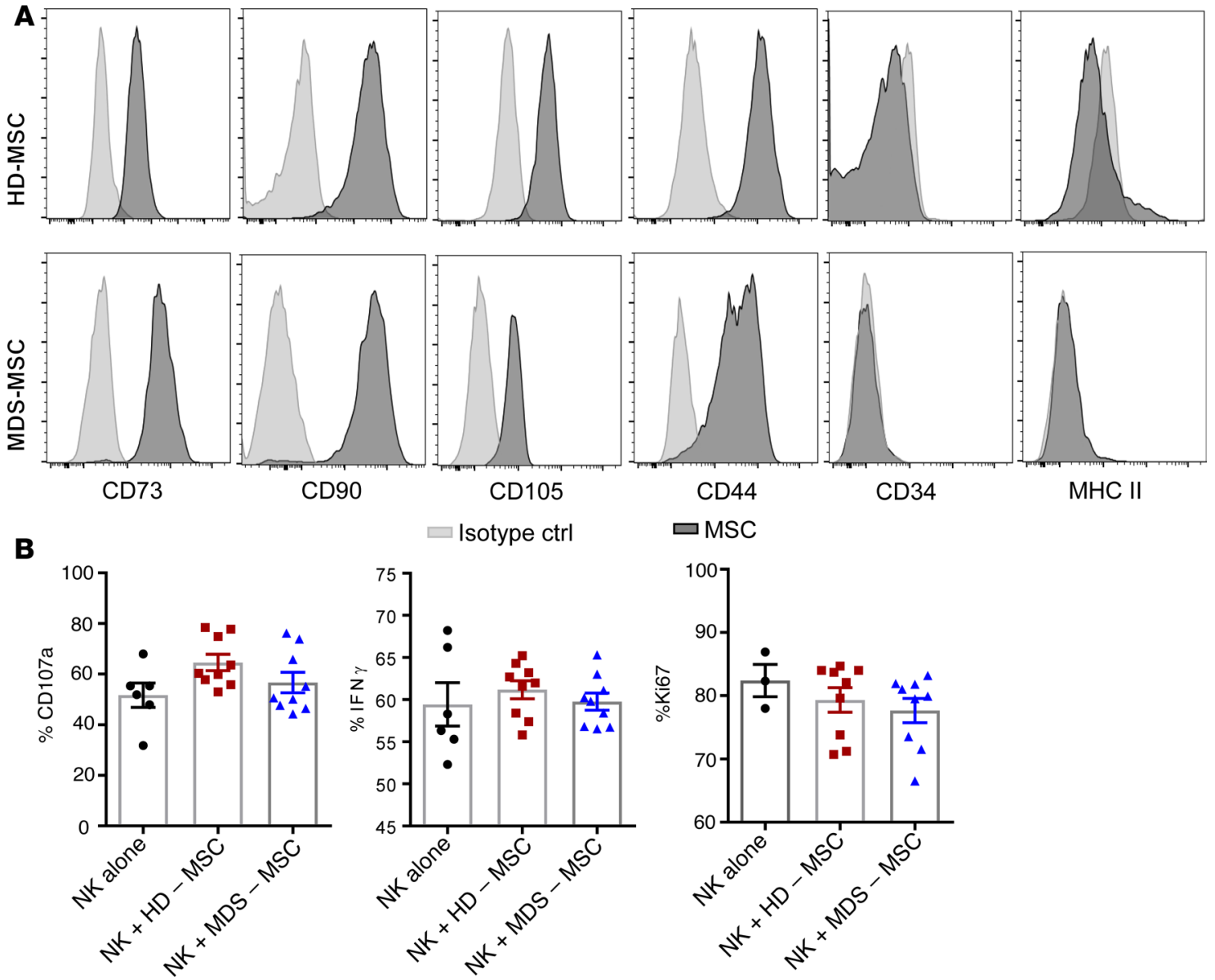

$\square$ Isotype ctrl

$\square$ MSC
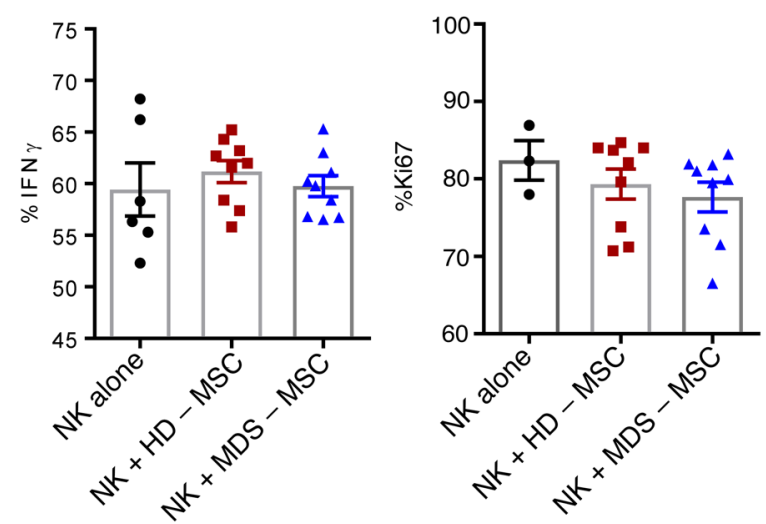

Figure 1. Healthy donor MSCs and MDS-MSCs are phenotypically similar and do not directly affect NK cell function. (A) Passage 3 healthy donor MSCs (HD-MSCs) ( $n=11)$ and MSCs from patients with MDS (MDS-MSCs) $(n=13)$ were stained for CD73, CD90, MHCII, CD105, and CD44 and evaluated by flow cytometry. Representative donors are shown. (B) NK cells were cultured directly with HD-MSCs and MDS-MSCs for 6 days in the presence of IL-15 (10 ng/ $\mathrm{ml}$ ) and stimulated with IL-2 and IL-18 and an anti-CD16 agonistic antibody 6 hours prior to analysis. NK cell function was evaluated by flow cytometry for degranulation (CD107a), IFN- $\gamma$ production, and proliferation (Ki67). Pooled data $(n=6-9)$ are shown as mean \pm SEM. Statistical analyses were performed using paired $t$ tests and, for multiple comparisons, were adjusted for significance using a FDR (FDR $<0.05$ ).

and 7-day MDS-MSC-conditioned monocytes. Hence, our data indicate that MDS-MSCs induce healthy monocytes to acquire a MDSC phenotype and metabolic capacity.

Monocytes cultured with MDS-MSCS but not HD-MSCS suppress NK and T cell function. MDSC accumulation in patients with MDS is associated with dysfunctional NK cells and CD8 ${ }^{+} \mathrm{T}$ cells correlating with poor prognosis $(24,26)$. To further explore this phenomenon, we next studied whether MDS-MSC cultured monocytes affect NK and T cell function compared with monocytes cultured with control HD-MSC. Purified NK cells and monocytes from the same heathy donor were cultured under various conditions for 4-6 days prior to assessing NK cell function. Neither 7-day control monocytes or 7-day HD-MSC-conditioned monocytes suppressed NK cell function. In marked contrast, 7-day MDS-MSC-conditioned monocytes inhibited NK cell degranulation, IFN- $\gamma$ and TNF- $\alpha$ production, and proliferation similar to that of NK cells cultured with in vitro-generated MDSCs (Figure 3A). This shows that HD-MSCs have no effect on monocytes, yet MDSMSCs induce monocytes to suppress the innate immune system.

$\mathrm{T}$ cell proliferation was next evaluated using allogeneic monocytes. Similar to NK cells, T cell proliferation was highly suppressed in the presence of 7-day MDS-MSC-conditioned monocytes compared with 7-day HD-MSC-conditioned monocytes or with 7-day control monocytes (Figure 3B). Thus, our results strongly suggest that MDS-MSCs induce the development of MDSCs from resting steady-state monocytes, indirectly inhibiting effector cell function.

MDS-MSCs and MDS-MSC-conditioned monocytes exhibit distinct genetic signatures compared with MSCs from healthy controls. Given the differential effects of MDS-MSCs on monocytes and (indirectly) on NK 
A

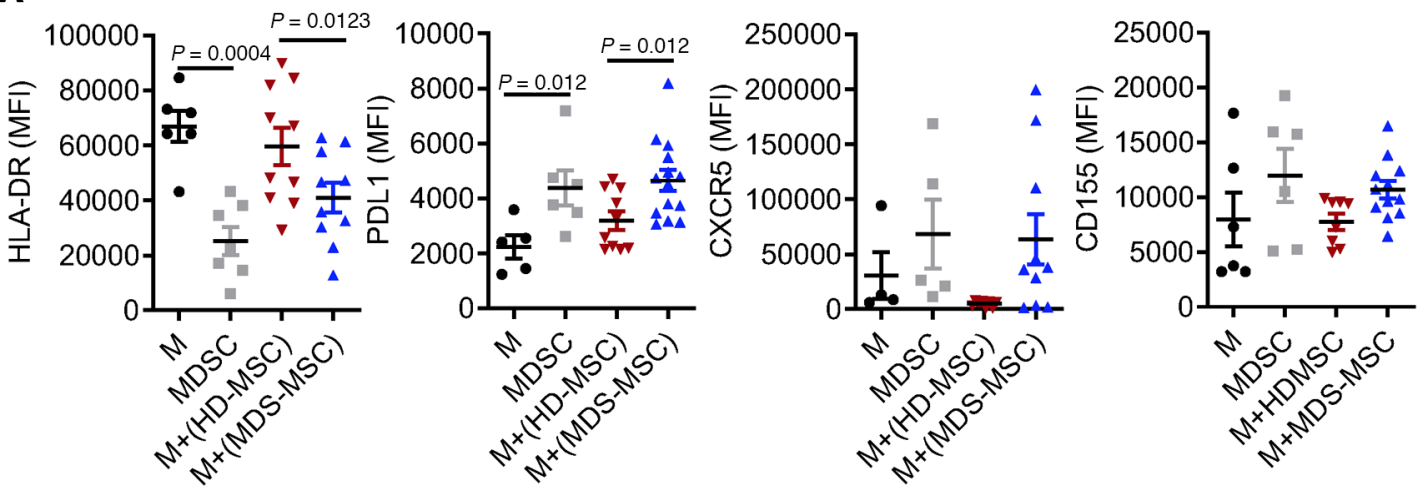

B

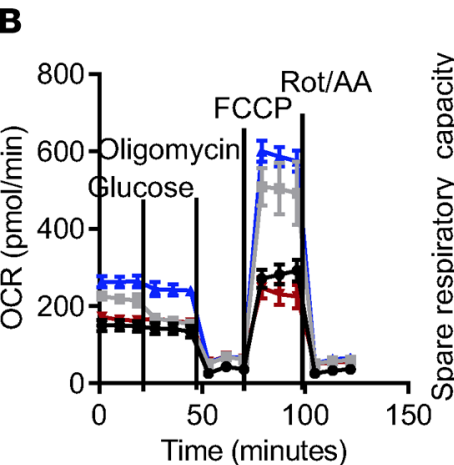

$\rightarrow$ Monocytes - MDSC

$\rightarrow \mathrm{M}+(\mathrm{HD}-\mathrm{MSC})-\mathrm{M}+(\mathrm{MDS}-\mathrm{MSC})$
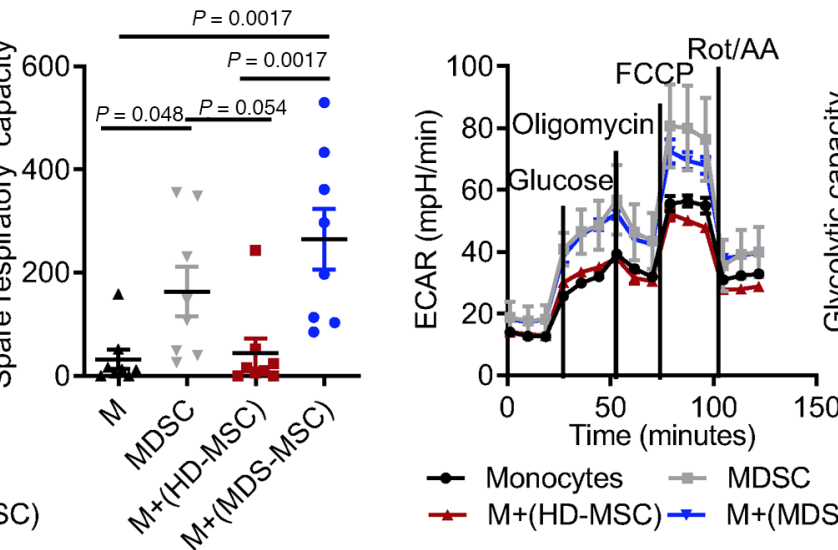

$\sim$ Monocytes $=$ MDSC

$\rightarrow \mathrm{M}+(\mathrm{HD}-\mathrm{MSC}) \rightarrow \mathrm{M}+(\mathrm{MDS}-\mathrm{MSC})$

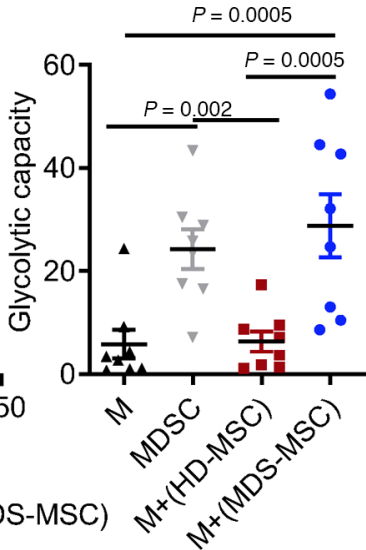

Figure 2. MDS-MSCs alter the phenotype and metabolic function of monocytes to resemble those of MDSCs. (A) Monocytes cultured with HD-MSCs or MDS-MSCs were evaluated by flow cytometry for the expression of HLA-DR, PD-L1, CXCR5, and CD155/PVR. Cumulative data from 5-11 donors are shown as mean \pm SEM. (B) Monocytes cultured with HD-MSCs $(n=8)$ and MDS-MSCs $(n=8)$ were cultured in 24-well plates and oxygen consumption rate (OCR) and the extracellular acidification rate (ECAR) were measured in real time in an XFe24 analyzer after injection of glucose, oligomycin, FCCP plus sodium pyruvate, and rotenone/antimycin A. Representative OCR and ECAR and cumulative mean \pm SEM data from spare respiratory capacity (SRC) and glycolytic capacity are shown. Paired $t$ tests were used for all comparisons, and, for multiple comparisons, FDR was used (FDR $<0.05)$.

cells, we hypothesized that these cells would have a distinct transcriptome compared with MSCs from HDs. To address this, we performed whole genome transcriptome analysis and analyzed the results with gene set enrichment analysis and Enrichr programs. Unsupervised genome analysis revealed homogeneity in MSC samples within patient groups and significant differential gene expression (DGE) between MDS and HDs (Figure 4A). Comparing MDS-MSCs to HD-MSCs, DGE revealed 660 genes (FDR, $P$ $\leq 0.01$ ). The top hundred genes included those involved in the regulation of immune-suppressive and innate immune pathways, including LIF, ENC1, TIMP3, IL-1R1, and IL-6, that are involved in myeloid suppressor cell function (Supplemental Table 1).

We next investigated genes in monocytes conditioned with MSCs. DGE of 7-day MDS-MSC-conditioned monocytes compared with 7-day HD-MSC-conditioned monocytes showed 25 genes (FDR, $P$ $\leq 0.01$ ) (Figure 4B). Analysis implicated DGE in the TGF- $\beta$, calcium, and glycolysis pathways (Figure 4C). Supervised analysis revealed that ENC1 and ALS2CL were increased in MDS-MSCs compared with HD-MSCs. In addition, monocytes conditioned with MDS-MSCs downregulated MAB21L2 (a downstream target of TGF- $\beta$ signaling) and upregulated MYOCD (a transcriptional coactivator of serum response factor [SRF]) and INHBA (TGF- $\beta$ superfamily member) (Figure 4D). These MSC-conditioned monocyte differences between HDs and patients with MDS suggest a mechanism of how MDS-MSCs contribute to a suppressive microenvironment.

MDS-MSC ROS production regulates TGF- $\beta$ production in monocytes. To confirm the expression changes found in the whole genome sequencing, we performed qRT-PCR to verify ENC1 and ALS2CL DGE in MDS-MSCs versus HD-MSCs. We showed increased expression of ENC1 in MDS-MSCs but not of 

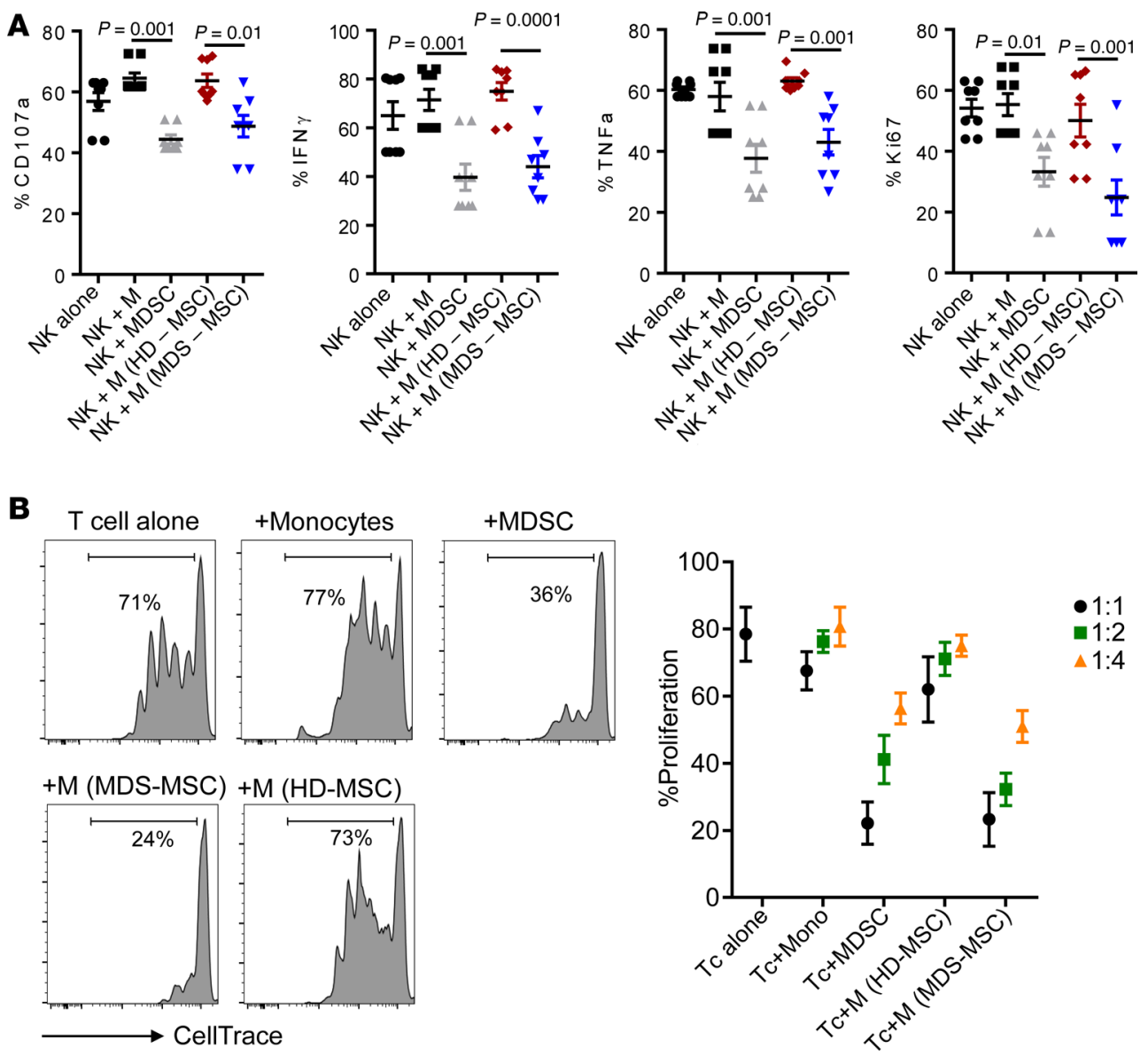

Figure 3. MDS-MSCs induce monocytes to suppress NK cell function and proliferation. (A) NK cells were cultured for 6 days with monocytes and HD-MSCs and MDS-MSCs in the presence of IL-15 $(10 \mathrm{ng} / \mathrm{ml})$ and stimulated with IL-12 and IL-18 and anti-CD16 6 hours prior staining. NK cell function was evaluated for degranulation (CD107a), IFN- $\gamma$ production, and TNF- $\alpha$ production, and proliferation (Ki67) was evaluated by flow cytometry. Pooled data $(n=8)$ are shown as mean \pm SEM. Statistical analyses were performed using 2-way ANOVA. (B) T cells were labeled with the proliferation dye CellTrace prior to coculture with monocytes precultured with HD-MSCs and MDS-MSCs. T cell cultures were stimulated with IL-15 (10 ng/ml) and CD3/CD28 for 3-4 days and T cell proliferation was evaluated by flow cytometry. One representative donor and pooled data $(n=6)$ are shown as mean \pm SEM.

ALS2CL at the transcript level; therefore, ALS2CL was not studied further (Figure 5A and Supplemental Figure 1D). In conditioned monocytes, MAB21L2 expression was significantly downregulated and INHBB expression was significantly upregulated in 7-day MDS-MSC-conditioned monocytes compared with 7-day HD-MSC-conditioned monocytes, confirming our transcriptome analysis (Figure 5B). To better understand the physiologic relevance in vivo, we investigated whether MAB21L2 was decreased in monocytes from the BM of patients with MDS compared with that of HDs. Confirming our in vitro experiments, we found that MAB21L2 expression was significantly lower in monocytes from HDs compared with that in MDS marrow monocytes (Figure 5C)

ENC1 is an inhibitor of the transcription factor Nrf2, which regulates ROS production and is thus hypothesized to regulate cellular stress levels (27). Therefore, we examined the production of ROS in MSCs. Cells were stimulated with or without hydrogen peroxide $\left(\mathrm{H}_{2} \mathrm{O}_{2}\right)$ for 1 hour and ROS was analyzed by flow cytometry. After $\mathrm{H}_{2} \mathrm{O}_{2}$ stimulation, MDS-MSCs produced 3-fold greater ROS compared with HD-MSCs $(n=4,4910 \pm 1400$ vs. $1500 \pm 330, P=0.05)$ (Figure 5D). ROS production has been associated with higher production of TGF- $\beta$ in MDSCs (28). In addition, TGF- $\beta$ production is counterbalanced by lower MAB21L2, a TGF- $\beta$ repressor. This results in a direct increase in monocyte production of soluble TGF- $\beta$ measured by intracellular staining after 7-day culture with MDS-MSCs (Figure 5E).

Cancer-derived MDSCs have also been shown to induce NK cell suppression through membrane-bound TGF- $\beta$ (29). Here, we investigated whether surface membrane-bound TGF- $\beta$ is also affect- 
A

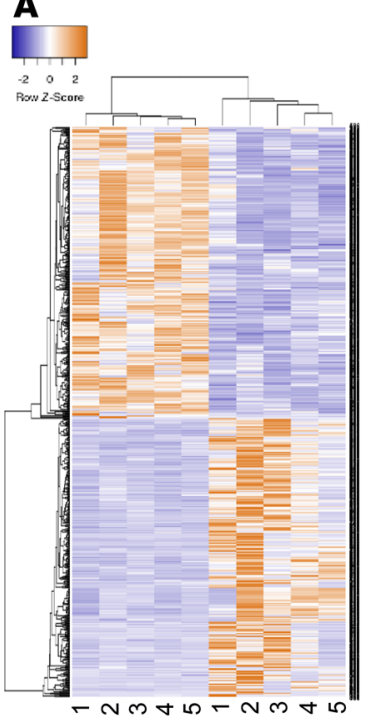

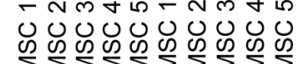

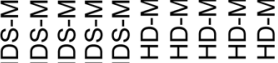

B

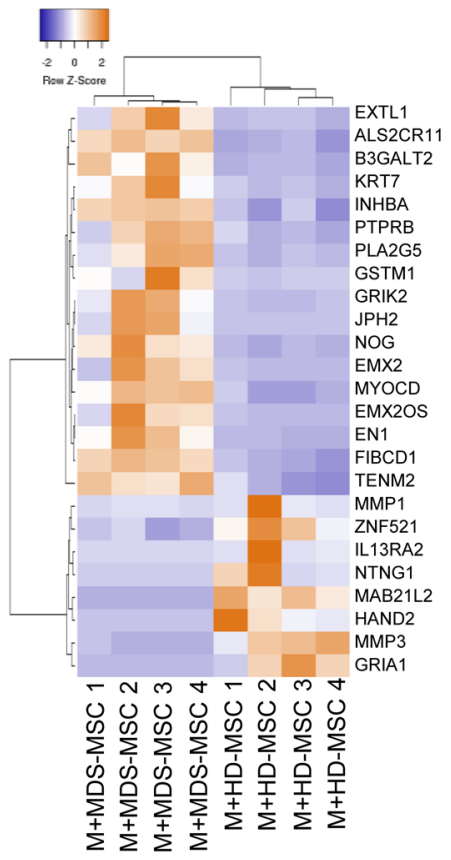

C

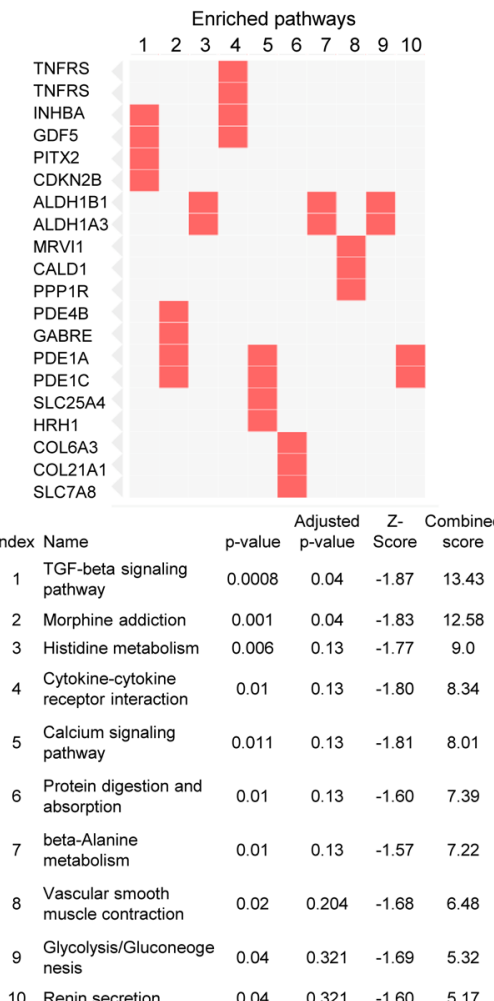

D
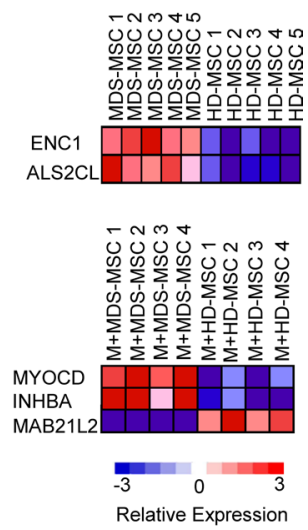

Relative Expression

Figure 4. Gene expression profile of MSCs and monocytes identifies putative gene differences between healthy donors and patients with MDS. Heatmap of the top differential gene expression (DEC) genes following RNA-sequencing of (A) HD-MSCs $(n=5)$ and MDS-MSCs $(n=5)$ or $(\mathbf{B})$ purified monocytes precultured with HD-MSCs and MDS-MSCs. (C) Pathway analysis performed by EnrichedR showed the positive enrichment of genes associated with top-ranked pathways involved in the regulation of monocytes cultured with MDS-MSCs compared with HD-MSCs. (D) The ranked DEC lists were used to perform gene set enrichment analysis using Gene Ontology annotated functions. Selected significantly altered gene set functions $(P \leq 0.01)$ show the contribution of individual genes to the enriched gene sets in HD-MSCs and MDS-MSCs or monocytes cultured with HD-MSCs and MDS-MSCs.

ed by the interaction with MDS-MSCs. Indeed, we found a significant increase in membrane-bound TGF- $\beta$ expression on 7-day MDS-MSC-conditioned monocytes compared with control cultures with HD-MSCs (Figure 5F). Supporting our in vitro studies, membrane-bound TGF- $\beta$ was increased on monocytes from MDS but not HD BM (Figure 5G).

We next investigated whether soluble or membrane-bound TGF- $\beta$ is responsible for inhibiting NK cell function. NK cells were cultured in Transwell inserts separated from MDS-MSC-conditioned monocytes, allowing for exchange of soluble factors but prohibiting direct cell-to-cell contact. Under these conditions, we found that MDS-MSC-conditioned monocytes were no longer able to suppress NK cells (Figure 6A). These data indicate that membrane-bound TGF- $\beta$ is likely part of the suppressive mechanism, but we cannot exclude other contact-mediated factors. In contrast to the contact-mediated effect of suppressive monocytes on NK cells, direct cell-to-cell contact was not needed for the conversion of monocytes to a suppressive phenotype by MDS-MSCs. These soluble factors induced suppressive monocytes, had similar phenotype as previously described (Figure 2A), and had the equal capacity to suppress NK cell function (Figure 6B). Cell-free supernatants containing only MDS-MSCderived exosomes could not induce a suppressive phenotype in HD monocytes (data not shown), suggesting that cell-to-cell interaction is needed for MSCs to regulate monocytes.

Knockdown of MSC ENC1 and the monocyte TGF- $\beta$ repressor MAB21L2 validates their role in the suppressive mechanism. To assess the potential involvement of the ENC1 gene in MSCs and the TGF- $\beta$ repressor MAB21L2 gene in monocytes in the immune-suppressive mechanism, we silenced each in the respective cell types using siRNA. Monocytes were conditioned for 7 days with MDS-MSCs transfected with negative control siRNA (scramble siRNA) or siRNA targeting ENC1 and assessed for TGF- $\beta$ production. When ENC1 was silenced in MDS-MSCs prior to culture with monocytes, we found a sharp decrease in mono- 
A

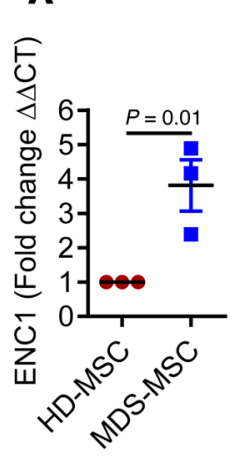

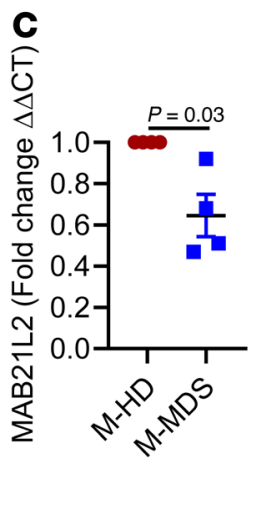

D
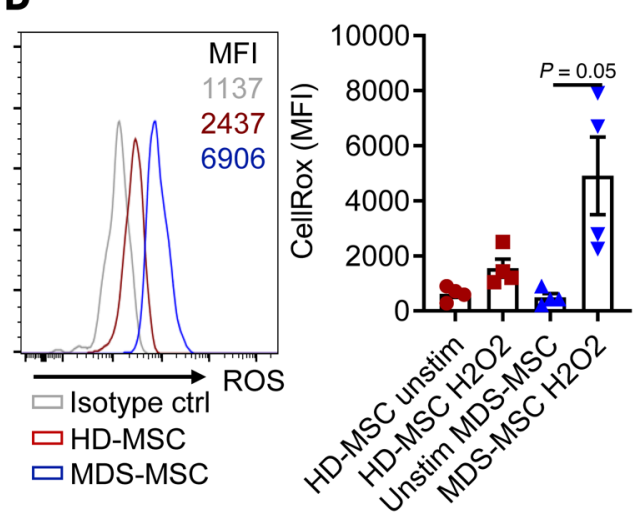

E

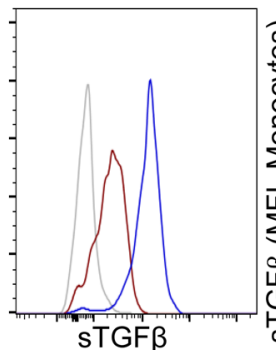

sTGF $\beta$

$\square$ Isotype ctrl

$\square$ HD-MSC

$\square$ MDS-MSC

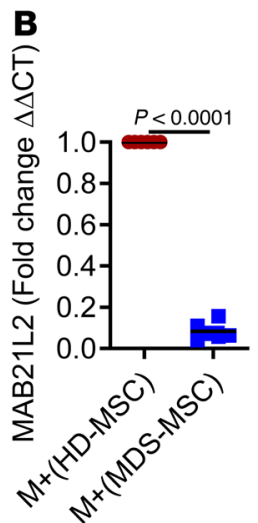

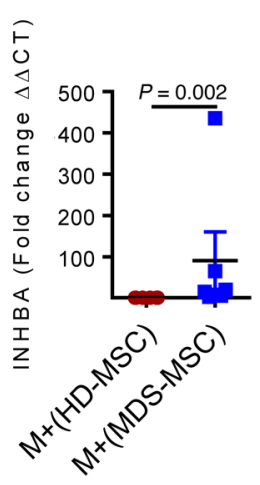

$\mathbf{F}$

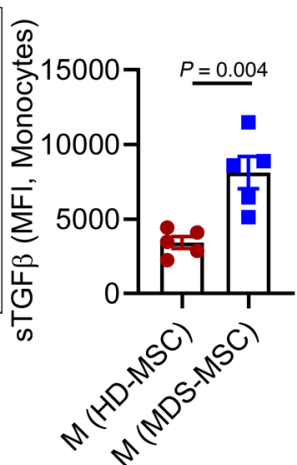

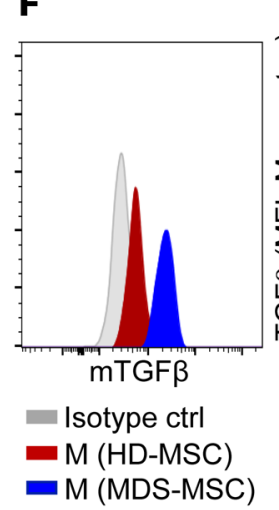

G

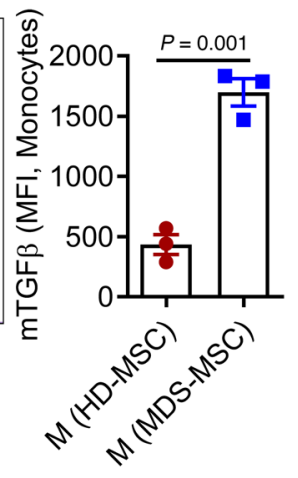

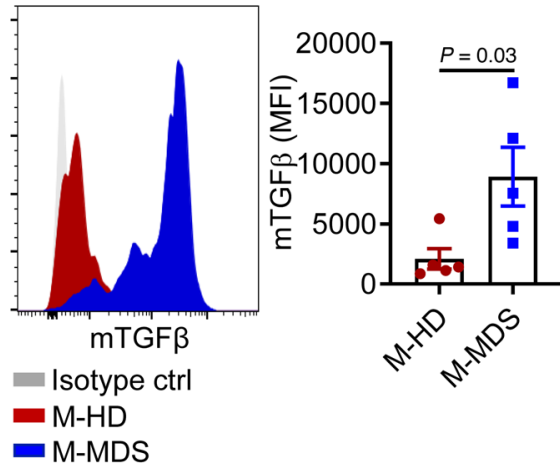

Figure 5. Quantitative PCR validates differences with ENC1 expression increases in MDC-MSCs and MAB21L2 expression decreases in monocytes exposed to MDS-MSCs, both of which lead to suppression mediated by TGF- $\beta$. Total RNA $(n=3-5)$ was isolated from monocytes alone, cytokine -derived MDSCs, monocytes precultured with HD-MSCs and MDS-MSCs, or HD-MSCs and MDS-MSCs, and the total mRNA was reverse transcribed into cDNA and analyzed by real-time quantitative PCR for (B) MAB21L2 and INHBA or (A) ENC1 gene expression. (C) Alternatively, RNA was isolated from MDS BM monocytes to evaluate MAB21L2 expression. Gene expression was normalized to the housekeeping gene GAPDH and calculated relative to control. (D) HD-MSCs and MDS-MSCs $(n=4)$ were analyzed by flow cytometry for total ROS following treatment with or without $\mathrm{H}_{2} \mathrm{O}_{2}(250 \mu \mathrm{M})$. (E) Monocytes cultured with HD-MSCs and MDS-MSCs were evaluated for intracellular TCF- $\beta$ (sTCF $\beta$ ). Representative and cumulative data $(n=5)$ are shown as mean \pm SEM. (F) Monocytes cultured with HD-MSCs and MDS-MSCs were evaluated for membrane-bound TGF- $\beta$ (mTCF $\beta$ ). Representative and cumulative data ( $n=3$ ) are shown as mean \pm SEM. (C) Cryopreserved BM mononuclear cells were cultured in medium overnight for recovery from freezing prior to CD14 cell isolation. Cells were stimulated with LPS for 6 hours prior to staining for mTCF $\beta$ expression in monocytes. Representative and cumulative data $(n=5)$ are shown as mean \pm SEM. Statistical analysis were performed using the Mann-Whitney $U$ test.

cyte TGF- $\beta$ production compared with that in those cultured with MDS-MSCs transfected with control siRNA (MFI, $34416 \pm 3180$ vs. $83470 \pm 1060$, Figure 6 C).

To mimic the MDS phenotype, we knocked down MAB21L2 gene expression in monocytes prior to HD-MSC conditioning. This resulted in an increased level of TGF- $\beta$ production (MFI, 11,673 $\pm 2380 \mathrm{vs}$. $3400 \pm 1120$, Figure $6 \mathrm{C}$ ), reproducing the MDS-MSC effect on monocytes. This definitively shows that MAB21L2 plays a central role in monocyte function that can be modulated by MDS-MSCs in the BM environment, promoting a suppressive phenotype mediated by an increase in TGF- $\beta$ production.

Having defined a role for MAB21L2 in the interaction between MDS-MSCs and monocytes, we investigated further to determine whether knockdown of MAB21L2 would validate its role in the NK cell-suppressive mechanism. Purified NK cells were cultured with monocytes that were conditioned with MDS-MSCs transfected with siRNA for ENC1 or control or monocytes transfected with siRNA for MAB21L2 following conditioning with HD-MSCs. After 5-6 days, they were analyzed for NK cell IFN- $\gamma$ production. Monocytes conditioned with ENC1-silenced MDS-MSCs blocked suppression, thus abrogating their inhibitory effect on NK cells. In addition, silencing the TGF- $\beta$ repressor MAB21L2 in normal monocytes following conditioning in culture with HD-MSCs induced suppressive function of monocytes, mimicking effects of diseased MDS-MSCs on monocytes, resulting in the inhibition of NK cell function (Figure 6D). 
A

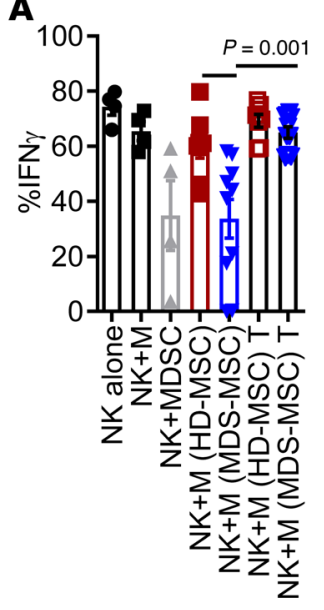

C

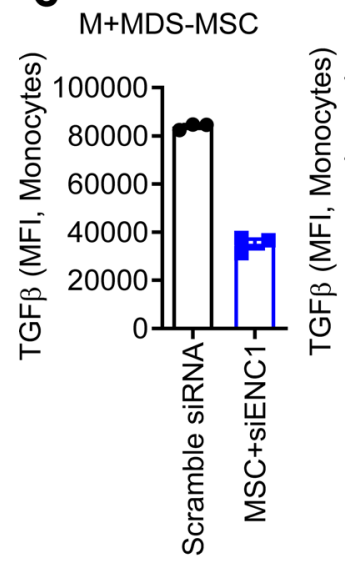

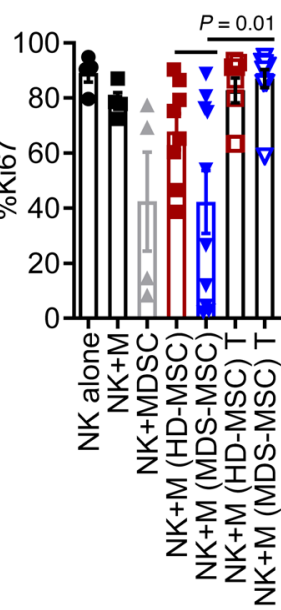

$\mathrm{M}+\mathrm{HD}-\mathrm{MSC}$

D
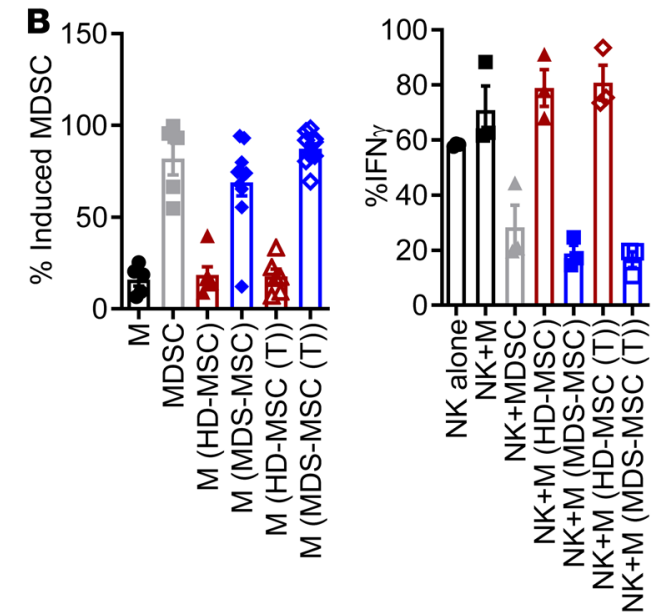

E
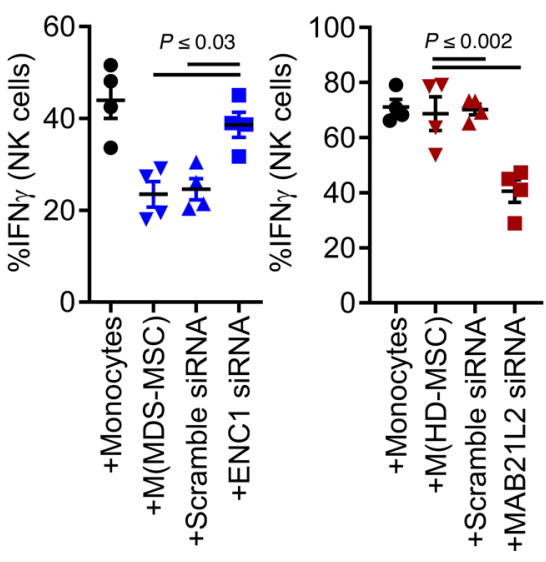

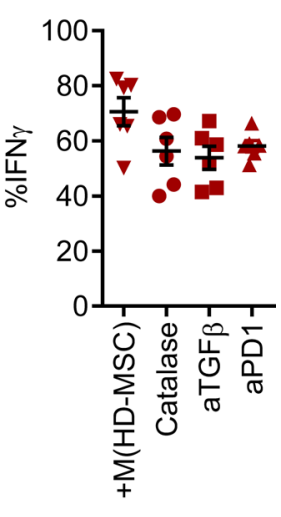

Figure 6. Knockdown of MDS-MSC ENC1 abrogates suppressive function and knockdown of MAB21L2 promotes recapitulates suppression function. (A) NK cells were cultured for 6 days with monocytes and HD-MSCs or MDS-MSCs $(n=8-10)$ in direct contact or separated by (T) Transwell inserts in the presence of IL-15 $(10 \mathrm{ng} / \mathrm{ml})$ and stimulated with IL-12 and IL-18 and anti-CD16 6 hours prior staining. NK cell function was evaluated for IFN- $\gamma$ production and proliferation (Ki67) by flow cytometry. (B) (Left) Monocytes were cultured with HD-MSCs and MDS-MSCs in direct contact or separated by Transwell

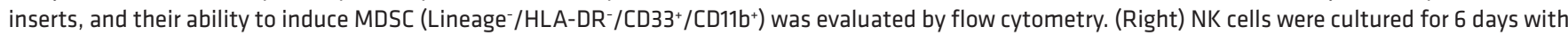
monocytes from cultures in the images to the left in direct contact in the presence of IL-15 (10 ng/ml) and stimulated with IL-12 and IL-18 and anti-CD16 6 hours prior staining. NK cell function was evaluated for IFN- $\gamma$ production by flow cytometry. Pooled data $(n=3)$ are shown as mean \pm SEM. (C) Monocytes $(n=3)$ were transfected with nontargeting or MAB21L2-targeting siRNA and cultured with HD-MSCs and evaluated for the production of TCF- $\beta$. Alternatively, monocytes $(n=3)$ were cocultured with MDS-MSCs transfected with nontargeting or ENC1-targeting siRNA and evaluated for the production of TGF- $\beta$. (D) NK cells $(n=4)$ were cultured with monocytes, monocytes cultured with MDS-MSCs for 5-6 days, and MDS-MSCs transfected with nontargeting or ENC1-targeting siRNA and NK cells. IFN- $\gamma$ production was evaluated by flow cytometry after 6 hours of stimulation with IL-12 and IL-18 and anti-CD16 prior to analysis. Additionally, NK cells were cultured with monocytes cultured with HD-MSCs and monocytes transfected with nontargeting or MAB21L2-targeting siRNA. (E) NK cells $(n=4)$ were cocultured with monocytes precultured with MDS-MSCs or HD-MSCs treated with catalase. Alternatively, NK cell monocyte cocultures were treated with anti-TGF- $\beta$ and anti-PD1, and NK cell IFN- $\gamma$ production was evaluated by flow cytometry after 6 hours of stimulation with anti-CD16 and IL-12 and IL-18. Pooled data are shown as mean \pm SEM, and statistical analyses were performed using 1-way ANOVA.

To confirm the dominant role of ENC1 and MAB21L2 expression in the suppressive mechanism, we blocked TGF- $\beta$, PD-1, and ROS production by antibodies or the ROS scavenger catalase for their effects on NK cell function. NK cell IFN- $\gamma$ production was restored to normal control levels in the presence of anti-TGF- $\beta$. Further, catalase treatment of 7-day MDS-MSC-conditioned monocytes prior to NK cell culture inhibited their suppression of NK cell function. PDL1 was upregulated in monocytes upon coculture with MDS-MSCs. However, blocking PD-1 when NK cells were cultured with 7-day MDSMSC-conditioned monocytes had no effect (Figure 6E). Control blocking experiments using NK cells and HD-MSC-conditioned monocytes had no effect on NK cell IFN- $\gamma$ production. Our data supports a mechanism where ROS produced by MDS-MSCs plays a critical role in NK cell suppression by transforming monocytes to MDSCs that produce TGF- $\beta$. 


\section{Discussion}

In this study, using primary MSCs isolated from patients with MDS and healthy BM, we showed that tumor-associated MSCs are responsible for inducing an immune-suppressive microenvironment in MDS through an indirect mechanism involving monocytes. MDS-MSCs exhibit a unique transcriptome with increased expression of the ROS pathway regulator gene ENC1, leading to a decrease in the TGF- $\beta$ repressor gene MAB21L2 in monocytes. These MDS-MSC-conditioned monocytes exerted strong inhibition of NK and T cell function, while HD-MSCs had no effect. The MDS-MSC functional phenotype was remarkably stable after 3-6 culture passages, suggesting acquired epigenetic regulation.

BM MSCs participate in the development of the hematopoietic niche and reconstruction of BM following tissue damage by inflammation and inflammatory effector cells. They display immunosuppressive properties, which may be lost in the absence of inflammatory stimuli (30). Indeed, we found that neither HD-MSCs nor MDS-MSCs directly inhibit NK cell function in vitro but MDS-MSCs work indirectly through monocytes.

We found that MDS-MSCs mediated a phenotypic and functional conversion of naive normal monocytes toward a suppressive MDSC-like phenotype, metabolic activity, and function. Consistent with these observations, Vasandan et al. elucidated a metabolic alteration of M1 proinflammatory (antitumor subtype) macrophages toward antiinflammatory M2 (protumor subtype) macrophages in a mechanism involving production of PGE2 (31). In addition, Galland and others have also highlighted that tumor-derived MSCs block NK cell activity in a direct interaction through production of PGE2 $(16,32)$. Although we found that monocytes become suppressor cells when exposed to MDS-MSCs, we did not observe a significant increase in PGE2 in our transcriptome analysis. The discrepancies between our data and others may be explained by the origin and location of MSCs and the environmental stimuli they receive. Alternatively, we might not have detected changes in PGE2 due to the timing of our gene expression analysis.

The observed shift of naive monocytes toward MDSCs following coculture with MDS-MSCs was consistent with a reduction in the expression of the TGF- $\beta$ repressor MAB21L2 and thus increased TGF- $\beta$ and functional immune suppression. In support of this, supervised analysis from the whole genome transcriptome further suggested a selective involvement of the TGF- $\beta$ pathway. Indeed, we found a robust increase in TGF- $\beta$ production in monocytes cocultured with MDS-MSCs. However, immune suppression of NK cells was abolished when they were separated from MDS-MSC-conditioned monocytes and only soluble factors could be exchanged, indicating that a membrane-bound protein is responsible for the functional inhibition. In fact, we found an increase in not only soluble TGF- $\beta$ but membrane-bound TGF- $\beta$ as well. In line with these findings, $\mathrm{Li}$ et al. found that tumor-expanded MDSCs induce functional anergy in NK cells through membrane-bound TGF- $\beta-1$ (33). Here, we found that TGF- $\beta$ production in monocytes was prompted by consistent induction of ROS in MDS-MSCs through upregulation of ENC1 expression. Cell-to-cell interaction between MDS-MSCs and monocytes was not required to induce MDSC characteristics in monocytes, supporting that a soluble factor is responsible for the induced changes in monocytes. ENC1 regulates cellular oxidative stress through the inhibition of Nrf2, which in turn negatively regulates ROS production (27). We have previously shown that the inhibitory MDS-MDSC mechanisms involve dysregulation of ROS (24). Chen et al. found that activation of the downstream pathway through Nrf 2 is responsible for increased TGF- $\beta$ production in MDS-MDSCs. Furthermore, a Nrf2-transgenic mouse model displayed BM accumulation of MDSCs accompanied by development of progressive multilineage dysplasia (34). These findings support the conclusion that ENC1-derived ROS enhances MDSC induction and regulation of their TGF- $\beta$ production.

In this study, knocking down ENC1 in MDS-MSCs diminished TGF- $\beta$ production in cocultured monocytes to abrogate immune suppression, directly implicating ENC1 in this mechanism. Additionally, silencing the TGF- $\beta$ repressor MAB21L2 in normal monocytes increased TGF- $\beta$ mimicking the suppressive function induced by MDS-MSCs to suppress NK and T cells $(29,35)$. Both ENC1 and MAB21L2 have documented roles in cancer progression. ENC1 and MAB21L2 dysregulation has recently been shown to be associated with a role of TGF- $\beta 1$ in the BM microenvironment of patients with MDS and AML (36). The malignant clone and MDS-MSCs can lead to both global alterations in hematopoiesis, increasing the risk of progression to AML. The resulting immunosuppressive microenvironment may contribute to MDS morbidity in part through the increased risk of infection seen in patients with MDS (37, 38). Yoshida et al. found increased mutations in ENC1 splicing machinery genes in MDS (84.9\%) and AML with myelodysplasia-related changes (25.8\%), suggesting an association of ENC1 with progression of MDS to AML (39). In two independent studies, researchers have uncovered a significant role of ENC1 in ovarian and colorectal cancer, where low expression indicates a favorable biomarker marker in ovarian cancer and 
upregulation of ENC1 is associated with colorectal carcinogenesis $(40,41)$. Together, these studies support our conclusion that the ENC1 gene in MDS-MSCs and MAB21L2 gene on monocytes play a crucial role in the progression of MDS.

Here, we focused on NK cell dysregulation in MDS and found that MDS-MSCs act indirectly by conversion of monocytes to MDSC-like cells to diminish NK cell activity. This at least partially explains the reduction in NK cell immune surveillance in patients with MDS $(42,43)$. There has been exponential growth in immune oncology toward boosting immune function to control disease relapse or, in the setting of MDS, preventing progression to AML. It is becoming increasingly clear that immune function is controlled in the microenvironment. The MSC mechanism identified here shows that immune suppression is indirectly communicated from the malignant clone to its supportive stroma. In this model, we propose that MDS-MSCs are not part of the malignant clone but that they are altered by either CD34 ${ }^{+}$or more differentiated cells originating from the malignant MDS clone (see graphical abstract). This "cancerized stroma" effect remains stable through several culture passages and is not a result of culture itself, as normal MSC controls had no effect on NK cell function. This model is supported by recent data from Poon et al. (44). They studied 6 patients with MDS with a karyotypic abnormality defining the MDS clone, and no karyotypic abnormalities were found in the MSCs from these subjects, supporting our proposed model. This supports the notion that the MDS malignant clone alters MSCs, which has functional consequences on immune function. Therapeutic strategies to normalize the suppressive marrow microenvironment in patients with MDS may be complicated, as they would have to spare the beneficial supportive role of the microenvironment in hematopoiesis. Future strategies to overcome NK cell immune suppression may be bypassed by NK cell adoptive transfer, activating cytokines, such as IL-15, or NK cell immune engagers that enhance immune-based therapies in MDS. These strategies will be the focus of future studies.

\section{Methods}

Patients and HDs. Cryopreserved MSCs from the BM of patients with MDS $(n=10)$ and BM from HDs obtained during hip replacement surgery $(n=11)$, as well as peripheral blood mononuclear cells from healthy blood donors, were obtained following Ficoll-Hypaque density gradient purification. Characteristics of patients with MDS and patients with healthy BM who underwent hip replacement are listed in Table 1 and Supplemental Table 2, respectively. Blood from HDs and BM samples from patients with MDS were procured from the Memorial Blood Bank (Minneapolis, Minnesota, USA) and the National Marrow Donor Program (NMDP)/Center for International Blood and Marrow Transplant Research Repository, respectively.

MSC isolation, culture, and conditioning of monocytes and metabolic function. MSCs were cultured initially in $\alpha$-MEM supplemented with 20\% FBS and subsequently with $10 \%$ FBS after removing nonadherent cells. Cultured MSCs were used in different experiments after 3-6 passages but not beyond to preserve genetic and phenotypic stability $(45,46)$.

Allogeneic monocytes were isolated from healthy blood donors by positive selection using CD14 magnetic beads and cultured alone (referred to as 7-day cultured control monocytes) or together with HD-MSCs or MDS-MSCs (referred to as 7-day MSC-conditioned monocytes) at a ratio of 5:1 with low-dose GM-CSF $(1 \mathrm{ng} / \mathrm{ml})$ in cell-to-cell contact or in a Transwell insert for 7 days of exposure. MDSCs were in vitro generated and characterized as previously described (47). A second positive selection of CD14 monocytes was performed for testing with $\mathrm{NK}$ and T cells.

Control monocytes and 7-day MSC-conditioned monocytes obtained after reisolation with magnetic beads were resuspended in glucose-free medium and rested overnight prior to Seahorse analysis (Seahorse XF Assay Medium, Agilent Technologies). Assays were carried out in 24-well plates with $0.25 \times 10^{6}$ cells per well. The ECAR and OCRs were measured (pmoles/min) in real time in an XFe24 analyzer (Agilent Technologies) after injection of glucose $(10 \mathrm{mM})$, oligomycin $(1 \mu \mathrm{M})$, FCCP $(1 \mu \mathrm{M})$ plus sodium pyruvate $(1 \mathrm{mM})$, and rotenone/ antimycin $\mathrm{A}(0.5 \mu \mathrm{M})$. The spare respiratory capacity was calculated from the change from basal oxygen consumption, after addition of glucose, to maximal oxygen consumption, after addition of FCCP. The glycolytic capacity was calculated from the change of maximal glycolysis subtracted from the basal ECAR.

$N K$ and $T$ cell function assays. Peripheral blood mononuclear cells were isolated from whole blood by density gradient centrifugation using Ficoll-Paque Premium (GE Healthcare). $\mathrm{CD}^{2} 6^{+} \mathrm{CD} 3^{-} \mathrm{NK}$ cells were isolated using negative depletion kits (EasySep Human NK Cell Enrichment Kit) (StemCell Technologies). $\mathrm{T}$ cells were isolated by $\mathrm{CD}^{+}$selecting microbeads. NK cells were cocultured with allogeneic MSCs for 6 days at a 5:1 ratio and evaluated for degranulation, IFN- $\gamma$ production, and proliferation. NK and T cell 
Table 1. Characteristics of patients with MDS

\begin{tabular}{|c|c|c|c|}
\hline Patient no. & Diagnosis & Age(yr) & Sex \\
\hline 1 & RAEB-2 & 36 & Male \\
\hline 2 & RAEB-2 & 13 & Male \\
\hline 3 & RARS & 68 & Male \\
\hline 4 & RARS & 66 & Male \\
\hline 5 & MDS & 65 & Female \\
\hline 6 & MDS/AML & 67 & Female \\
\hline 7 & REAB & 66 & Female \\
\hline 8 & RAEB-2 & 72 & Male \\
\hline 9 & RAEB & 65 & Female \\
\hline 10 & RARS & 53 & Male \\
\hline
\end{tabular}

RAEB, refractory anemia with excess blasts; RARS, refractory anemia with ringed sideroblasts; MDS, myelodysplastic syndrome; AML, acute myelogenous leukemia.

function was assessed after incubation with control monocytes and 7-day MSC-conditioned monocytes at a 1:1 ratio, in direct cell-to-cell contact or in a Transwell insert allowing for soluble factor exchange only, in RPMI supplemented with IL-15 (10 ng/ml) for 5-6 days for NK cells. NK cell function was then evaluated following stimulation with anti-CD16 $(1 \mu \mathrm{g} / \mathrm{ml}), \mathrm{IL}-12(5 \mathrm{ng} / \mathrm{ml})$, and IL-18 $(50 \mathrm{ng} / \mathrm{ml})$ for 6 hours prior to staining. T cell proliferation was performed by labeling with CellTrace $(5 \mu \mathrm{M})$ and stimulation using CD3/ CD28 activation beads and IL-15 $(10 \mathrm{ng} / \mathrm{ml})$ in a mixed lymphocyte reaction.

RNA-sequencing. HD-MSCs and MDS-MSCs and bead-purified 7-day control or MSC-conditioned monocytes were cultured as indicated. RNA was purified using a RNeasy Plus Micro Kit (Qiagen) to a final volume of $14 \mu 1$ and stored in RLT buffer (Qiagen) at $-80^{\circ} \mathrm{C}$. cDNA was generated using the Clonetech SMARTer Kit (Clonetech). Barcoded TruSeq RNA v2 libraries (Illumina) were created and pooled libraries were loaded onto High-Output flow cells and sequenced using a HiSeq2500 System (Illumina) and 50-bp paired-end reads. Raw sequencing FASTQ files were assessed for quality control using FASTQC software (http://www.bioinformatics.babraham.ac.uk/projects/fastqc/). Reads were mapped to the GRCh37 human reference genome with the STAR algorithm v2.5.0a.24. Raw read counts were output into $\mathrm{R}$ (version 3.2.0) and subjected to normalization by the trimmed mean of $\mathrm{M}$ values normalization method implemented in the $\mathrm{R} /$ bioconductor edge $\mathrm{R}$ package and variance normalized using voom from the R/bioconductor limma package. All genes with at least 1 count per million mapped reads in at least 2 samples were analyzed further. DGE was assessed using the R/ bioconductor limma package. Fold change and FDR $<0.05$ are reported. Heatmaps were generated for genes using R programming. Enriched pathways were evaluated using gene set enrichment analysis and Enrichr (48-51).

Quantitative RT-PCR. For quantification of gene expression, RNA was isolated from MSCs and purified monocytes from blood or marrow of HDs or patients with MDS using a RNeasy Micro Kit or Rneasy Mini Kit (Qiagen). cDNA was synthesized from RNA using Superscript IV Reverse transcription (Thermo Fisher) $\left(37^{\circ} \mathrm{C}\right.$ for 15 minutes, $65^{\circ} \mathrm{C}$ for 10 minutes). qRT-PCR reactions were performed using TaqMan Universal Master Mix (Thermo Fisher). Primers used for analysis of gene expression and mRNA quantification are for INHBA (Hs00173582_m1), MAB21L2 (Hs01040900_s1), ENC1 (Hs00171580_m1), ALS2CL (Hs00377660_m1), and GAPDH (Hs02758991_g1). All reactions were carried out in the Bio-Rad thermal cycler and Applied Biosystems 7500 Real Time PCR System.

Flow cytometry analysis. For phenotype analyses, BM monocytes, MSCs, and 7-day control or MSC-conditioned monocytes were examined for MSC marker expression of CD73, CD90, MHCII, CD105, and CD44 or monocyte protein expression of CD11b, HLA-DR, PDL1, CXCR5, CXCR2, nectin-2, CD155, and membrane-bound TGF- $\beta$. Subsequently, monocyte, MSC, and NK cell function was assessed by intracellular staining using fluorochrome-conjugated antibodies or reactive dye against ROS, TGF- $\beta$, IFN- $\gamma$, TNF- $\alpha$, Ki67 (proliferation), and CD107a (degranulation). Detection of cytokines, CD107a, and Ki67 was performed following fixation and permeabilization (eBioscience) according to the manufacturer's instructions. All cells were acquired by LSRII (BD Biosciences) and analyzed by FlowJo 10.0. 
MSC and monocyte siRNA transfection. MSCs were seeded in 6-well plates and overnight were transfected with Lipofectamine RNAiMAX transfecting agent (Thermo Fisher) containing control Silencer Select Small interfering RNA (siRNA) (Thermo Fisher) (10 nM) or siRNA for ENC1 (10 nM) 1 day prior to MSC conditioning of monocytes. In separate experiments on 7-day HD-MSC-conditioned monocytes prior to culture with NK cells, conditioned monocytes were seeded in 6-well plates and overnight were transfected with siRNA for MAB21L2 and control, according to the manufacturer's recommendations (Thermo Fisher). Transfection efficacy was analyzed by mRNA expression in qRT-PCR.

Data sharing Statement. The RNA-sequencing data were deposited in the NCBI's Gene Expression Omnibus database (GEO GSE140101).

Statistics. All data were analyzed using software cited above and summarized by Prism software (version 7 , GraphPad). All data were first tested for normal distribution. Thereafter, differences and correlations among groups were analyzed by 2-tailed Student's $t$ test and 1- or 2-way ANOVA (as indicated in the figure legends) and Mann-Whitney $U$ test. $P$ values were corrected using FDR for multiple comparisons (FDR $<0.05$ was considered significant). Representative histograms or images were chosen based on the average values.

Study approval. All samples were deidentified and their use was approved by the University of Minnesota and NMDP institutional review boards, in accordance with the Declaration of Helsinki.

\section{Author contributions}

DS, JW, USA, and CH performed experiments and analyzed data. DS, MRV, BG, EW, and BRB interpreted data and wrote the paper. JSM supervised design, analysis, and study interpretation and wrote the paper.

\section{Acknowledgments}

We would like to thank the University of Minnesota Genomics Center and Juan E. Abrahante Lloréns for performing the whole genome sequencing and running the preliminary analysis. We would also like to thank Michelle Roesler for her efforts to identify MDS samples for this study. This work was supported by National Institutes of Health grants P01 CA65453 (to JSM and BRB), P01 CA111412 (to JSM), R35 CA197292 (to JSM), and R01 HL56067 (to BRB) and the Karolinska Institutet through a fellowship to DS.

Address correspondence to: Jeffrey S. Miller, University of Minnesota, 420 Delaware Street SE, MMC 806, Minneapolis, Minnesota 55455, USA. Phone: 612.625.7409; Email: mille011@umn.edu.

1. Bejar R, et al. Clinical effect of point mutations in myelodysplastic syndromes. N Engl J Med. 2011;364(26):2496-2506.

2. Papaemmanuil E, et al. Clinical and biological implications of driver mutations in myelodysplastic syndromes. Blood. 2013;122(22):3616-3627.

3. Huang JC, et al. Mesenchymal stromal cells derived from acute myeloid leukemia bone marrow exhibit aberrant cytogenetics and cytokine elaboration. Blood Cancer J. 2015;5:e302.

4. Poon Z, et al. Bone marrow MSCs in MDS: contribution towards dysfunctional hematopoiesis and potential targets for disease response to hypomethylating therapy. Leukemia. 2019;33(6):1487-1500.

5. Rivera-Cruz CM, Shearer JJ, Figueiredo Neto M, Figueiredo ML. The immunomodulatory effects of mesenchymal stem cell polarization within the tumor microenvironment niche. Stem Cells Int. 2017;2017:4015039.

6. Corradi G, et al. Mesenchymal stromal cells from myelodysplastic and acute myeloid leukemia patients display in vitro reduced proliferative potential and similar capacity to support leukemia cell survival. Stem Cell Res Ther. 2018;9(1):271.

7. Rathnayake AJ, Goonasekera HW, Dissanayake VH. Phenotypic and cytogenetic characterization of mesenchymal stromal cells in de novo myelodysplastic syndromes. Anal Cell Pathol (Amst). 2016;2016:8012716.

8. Kastrinaki MC, Pontikoglou C, Klaus M, Stavroulaki E, Pavlaki K, Papadaki HA. Biologic characteristics of bone marrow mesenchymal stem cells in myelodysplastic syndromes. Curr Stem Cell Res Ther. 2011;6(2):122-130.

9. Pittenger MF, et al. Multilineage potential of adult human mesenchymal stem cells. Science. 1999;284(5411):143-147.

10. Oswald J, et al. Mesenchymal stem cells can be differentiated into endothelial cells in vitro. Stem Cells. 2004;22(3):377-384

11. Makino S, et al. Cardiomyocytes can be generated from marrow stromal cells in vitro. J Clin Invest. 1999;103(5):697-705.

12. Snykers S, De Kock J, Rogiers V, Vanhaecke T. In vitro differentiation of embryonic and adult stem cells into hepatocytes: state of the art. Stem Cells. 2009;27(3):577-605.

13. Gao F, et al. Mesenchymal stem cells and immunomodulation: current status and future prospects. Cell Death Dis. 2016;7:e2062.

14. Jiang XX, et al. Human mesenchymal stem cells inhibit differentiation and function of monocyte-derived dendritic cells. Blood. 2005;105(10):4120-4126.

15. Beyth S, et al. Human mesenchymal stem cells alter antigen-presenting cell maturation and induce T-cell unresponsiveness. Blood. 2005;105(5):2214-2219.

16. Spaggiari GM, Capobianco A, Abdelrazik H, Becchetti F, Mingari MC, Moretta L. Mesenchymal stem cells inhibit natural killer-cell proliferation, cytotoxicity, and cytokine production: role of indoleamine 2,3-dioxygenase and prostaglandin E2. Blood. 2008;111(3):1327-1333. 
17. Gao WX, et al. Effects of mesenchymal stem cells from human induced pluripotent stem cells on differentiation, maturation, and function of dendritic cells. Stem Cell Res Ther. 2017;8(1):48.

18. Aggarwal S, Pittenger MF. Human mesenchymal stem cells modulate allogeneic immune cell responses. Blood. 2005;105(4):1815-1822.

19. Sato K, et al. Nitric oxide plays a critical role in suppression of T-cell proliferation by mesenchymal stem cells. Blood. 2007;109(1):228-234.

20. Di Nicola M, et al. Human bone marrow stromal cells suppress T-lymphocyte proliferation induced by cellular or nonspecific mitogenic stimuli. Blood. 2002;99(10):3838-3843.

21. Dominici M, et al. Minimal criteria for defining multipotent mesenchymal stromal cells. The International Society for Cellular Therapy position statement. Cytotherapy. 2006;8(4):315-317.

22. Gao F, et al. Mesenchymal stem cells and immunomodulation: current status and future prospects. Cell Death Dis. 2016;7:e2062.

23. Lin W, Xu L, Zwingenberger S, Gibon E, Goodman SB, Li G. Mesenchymal stem cells homing to improve bone healing. J Orthop Translat. 2017;9:19-27.

24. Sarhan D, et al. Adaptive NK cells with low TIGIT expression are inherently resistant to myeloid-derived suppressor cells. Cancer Res. 2016;76(19):5696-5706.

25. Jian SL, et al. Glycolysis regulates the expansion of myeloid-derived suppressor cells in tumor-bearing hosts through prevention of ROS-mediated apoptosis. Cell Death Dis. 2017;8(5):e2779.

26. Shao ZH, Jiang HJ, Fu R. Increased population of myeloid-derived suppressor cells in patients with myelodysplastic syndromes overexpress ARG1 and mediate CD8(+) T cell inhibition. Blood. 2013; 122(21):5212.

27. Wang XJ, Zhang DD. Ectodermal-neural cortex 1 down-regulates Nrf2 at the translational level. PLoS One. 2009;4(5):e5492.

28. Zhang $\mathrm{H}$, et al. Myeloid-derived suppressor cells inhibit $\mathrm{T}$ cell proliferation in human extranodal NK/T cell lymphoma: a novel prognostic indicator. Cancer Immunol Immunother. 2015;64(12):1587-1599.

29. Li H, Han Y, Guo Q, Zhang M, Cao X. Cancer-expanded myeloid-derived suppressor cells induce anergy of NK cells through membrane-bound TGF-beta 1. J Immunol. 2009;182(1):240-249.

30. Spaggiari GM, Moretta L. Cellular and molecular interactions of mesenchymal stem cells in innate immunity. Immunol Cell Biol. 2013;91(1):27-31.

31. Vasandan AB, Jahnavi S, Shashank C, Prasad P, Kumar A, Prasanna SJ. Human Mesenchymal stem cells program macrophage plasticity by altering their metabolic status via a PGE 2 -dependent mechanism. Sci Rep. 2016;6:38308.

32. Galland S, et al. Tumor-derived mesenchymal stem cells use distinct mechanisms to block the activity of natural killer cell subsets. Cell Rep. 2017;20(12):2891-2905.

33. Li H, Han Y, Guo Q, Zhang M, Cao X. Cancer-expanded myeloid-derived suppressor cells induce anergy of NK cells through membrane-bound TGF-beta 1. J Immunol. 2009;182(1):240-249.

34. Chen X, et al. Induction of myelodysplasia by myeloid-derived suppressor cells. J Clin Invest. 2013;123(11):4595-4611

35. Gabrilovich DI, Nagaraj S. Myeloid-derived suppressor cells as regulators of the immune system. Nat Rev Immunol. 2009;9(3):162-174.

36. Geyh S, et al. Transforming growth factor $\beta 1$-mediated functional inhibition of mesenchymal stromal cells in myelodysplastic syndromes and acute myeloid leukemia. Haematologica. 2018;103(9):1462-1471.

37. Warlick ED, Miller JS. Myelodysplastic syndromes: the role of the immune system in pathogenesis. Leuk Lymphoma. 2011;52(11):2045-2049.

38. Steensma DP. Myelodysplastic syndromes current treatment algorithm 2018. Blood Cancer J. 2018;8(5):47.

39. Yoshida K, et al. Frequent pathway mutations of splicing machinery in myelodysplasia. Nature. 2011;478(7367):64-69.

40. Fan S, et al. Low expression of ENC1 predicts a favorable prognosis in patients with ovarian cancer. J Cell Biochem. 2019;120(1):861-871.

41. Fujita M, Furukawa Y, Tsunoda T, Tanaka T, Ogawa M, Nakamura Y. Up-regulation of the ectodermal-neural cortex 1 (ENC1) gene, a downstream target of the beta-catenin/T-cell factor complex, in colorectal carcinomas. Cancer Res. 2001;61(21):7722-7726.

42. Sarhan D, et al. 161533 TriKE stimulates NK-cell function to overcome myeloid-derived suppressor cells in MDS. Blood Adv. 2018;2(12):1459-1469.

43. Gleason MK, et al. CD16xCD33 bispecific killer cell engager (BiKE) activates NK cells against primary MDS and MDSC CD33+ targets. Blood. 2014;123(19):3016-3026.

44. Poon Z, et al. Correction: Bone marrow MSCs in MDS: contribution towards dysfunctional hematopoiesis and potential targets for disease response to hypomethylating therapy. Leukemia. 2019;33(6):1542.

45. Gatto F, et al. Hurler disease bone marrow stromal cells exhibit altered ability to support osteoclast formation. Stem Cells Dev. 2012;21(9):1466-1477.

46. Medyouf $\mathrm{H}$, et al. Myelodysplastic cells in patients reprogram mesenchymal stromal cells to establish a transplantable stem cell niche disease unit. Cell Stem Cell. 2014;14(6):824-837.

47. Sarhan D, Miller JS. Assessing canonical and adaptive natural killer cell function in suppression assays in vitro. Methods Mol Biol. 2019;1913:153-166.

48. Subramanian A, et al. Gene set enrichment analysis: a knowledge-based approach for interpreting genome-wide expression profiles. Proc Natl Acad Sci USA. 2005;102(43):15545-15550.

49. Mootha VK, et al. PGC-1alpha-responsive genes involved in oxidative phosphorylation are coordinately downregulated in human diabetes. Nat Genet. 2003;34(3):267-273.

50. Chen EY, et al. Enrichr: interactive and collaborative HTML5 gene list enrichment analysis tool. BMC Bioinformatics. 2013; $14: 128$.

51. Kuleshov MV, et al. Enrichr: a comprehensive gene set enrichment analysis web server 2016 update. Nucleic Acids Res. 2016:44(W1):W90-W97. 